\title{
Current outcomes of postrecurrence survival in patients after resection of non-small cell lung cancer
}

\author{
Tetsuya Mizuno ${ }^{1}$, Takaaki Arimura ${ }^{1}$, Hiroaki Kuroda ${ }^{1}$, Noriaki Sakakura ${ }^{1}$, Yasushi Yatabe ${ }^{2}$, Yukinori \\ Sakao \\ ${ }^{1}$ Division of Thoracic Surgery, ${ }^{2}$ Division of Pathology and Molecular Diagnostics, Aichi Cancer Center Hospital, Nagoya, Japan \\ Contributions: (I) Conception and design: T Mizuno, Y Sakao; (II) Administrative support: Y Sakao; (III) Provision of study materials or patients: \\ Y Sakao, Y Yatabe; (IV) Collection and assembly of data: T Mizuno, T Arimura H Kuroda, N Sakakura; (V) Data analysis and interpretation: T \\ Mizuno, Y Sakao; (VI) Manuscript writing: All authors; (VII) Final approval of manuscript: All authors. \\ Correspondence to: Tetsuya Mizuno, MD. Division of Thoracic Surgery, Aichi Cancer Center Hospital, Kanokoden1-1, Chikusa-ku, Nagoya 464-8681, \\ Japan. Email: t-mizuno@aichi-cc.jp.
}

Background: Despite complete resection, patients with resectable non-small cell lung cancer (NSCLC) sometimes experience recurrence in various sites, generally with an unfavorable prognosis. Receptor tyrosine kinase inhibitors (TKIs) have produced a dramatic response in patients with advanced NSCLC harboring sensitive mutations.

Methods: We retrospectively investigated 281 patients with recurrence who underwent complete resection of their NSCLC between January 2005 and December 2013 with tumors in which the status of epidermal growth factor receptor (EGFR) mutation, KRAS mutation, and anaplastic lymphoma kinase (ALK) rearrangement was confirmed. Clinicopathological factors, including mode of recurrence, oncogenic status, and postrecurrence survival (PRS), were reviewed. We aimed to investigate the prognostic factors of PRS by univariate and multivariate analyses.

Results: Among the 281 patients, 135 patients (48.0\%) with EGFR mutation, 33 (11.7\%) with KRAS mutation, 13 (4.7\%) with ALK rearrangement, and 100 (35.6\%) with triple negative (TN) mutation were identified. Median survival time after recurrence was 26.1 months. In multivariate analysis, the presence of EGFR mutation, pStage I, the presence of both local and systemic therapies for recurrence, and longer time to recurrence (TTR) were significant favorable factors for PRS. With regard to the initial site of recurrence, the presence of pleural and/or bone recurrence reduced PRS significantly. The presence of pulmonary recurrence increased PRS, especially in patients with EGFR- or ALK-mutated tumors.

Conclusions: This study documented the current outcomes of PRS. EGFR mutated status, pStage I, longer TTR and presence of multimodal therapy for recurrence were favorable factors for PRS.

Keywords: Postoperative recurrence; initial recurrent site; oncogenic status

Submitted Nov 09, 2017. Accepted for publication Jan 09, 2018.

doi: $10.21037 /$ jtd.2018.01.148

View this article at: http://dx.doi.org/10.21037/jtd.2018.01.148

\section{Introduction}

Lung cancer is a leading cause of cancer deaths worldwide, including Japan. In this decade, biological and molecular profiling of lung cancer has enabled precise treatment for advanced disease. For example, cytotoxic chemotherapy with pemetrexed improved survival in patients with advanced non-small cell lung cancer (NSCLC) of nonsquamous histology (1). Molecular targeted therapy also produced favorable outcomes in selected patients. Epidermal growth factor receptor (EGFR) gene mutations are identified most commonly as oncogenic driver mutations in Asian patients with NSCLC, and 70-80\% of tumors with these mutations responded to small-molecule tyrosine kinase inhibitors 
(TKIs) targeting EGFR (2-5). Anaplastic lymphoma kinase (ALK) inhibitors produced good responses and prognostic outcomes in tumors with ALK rearrangement $(6,7)$.

For patients with early or locally advanced disease, surgical resection is initially indicated as an option for radical treatment. However, approximately half of patients undergoing resection experience recurrence in locoregional and/or distant sites (8-12). Patients with recurrence after resection are often treated by the same systemic therapy as patients with advanced disease, but their prognostic outcomes may not be identical (13-15). Although treatment strategy varies according to the oncogenic status of the tumor and the mode of recurrence, the effects of current strategies on postrecurrence survival (PRS) are not well understood.

In this study, we aimed to investigate PRS in patients with resected NSCLC in association with oncogenic status of the tumors and patterns of recurrence. We also aimed to identify the factors associated with PRS.

\section{Methods}

From January 2005 through December 2013, 1,305 patients underwent resection of NSCLC at Aichi Cancer Center Hospital. Patients with R1/2 resection were excluded from the study. Three hundred fifty-three patients $(27.0 \%)$ had a recurrence after resection. Among these, we identified 281 patients with tumors in which the status of EGFR mutation, KRAS mutation, and ALK rearrangement was confirmed, and we included them in the study. The median follow-up period for the 281 patients after recurrence was 26.1 months (range, 1.3-108.3 months), and that of surviving patients was 29.6 months (range, 1.4-85.8 months).

Clinicopathological data regarding age, gender, smoking history, histology, pathological nodal status, surgical procedure, oncogenic status, time to recurrence (TTR), treatment for recurrence, and PRS were reviewed from the medical records. For further analyses of the effect of metastatic site on PRS, the records of the initial metastatic site were also collected. Primary tumor samples were obtained from all 281 patients. Mutational analysis of oncogenes was performed as previously described (16). In brief, reverse transcriptase-polymerase chain reaction direct sequencing of EGFR, KRAS, and ALK was used to assess the mutational status in cases with available frozen tissues. In the case with available paraffin-embedded tissues, mutational status was determined using cycleave methods for EGFR and KRAS. ALK rearrangement was screened using immunohistochemistry, and fluorescence in situ hybridization was used for confirmation of ALK rearrangement.

Chest CT every 6 months until 2 years postoperatively and once a year thereafter were used as the standard followup protocol. PET scan and brain surveillance were not mandatory; introductions of them depended on physicians. The initial sites of recurrence were defined as the recurrent sites diagnosed first after resection, other sites identified in subsequent systemic surveillance, as well as those diagnosed within 1 month from the initial detection.

The study was approved by our institutional review board and waived the need for written informed consent from each patient.

PRS was measured from the date of initial recurrence until either death or the last day of follow-up. Survival curves were created by the Kaplan-Meier method. Univariate and multivariate analyses were performed using the Cox regression hazard model to assess the prognostic significance of the individual factors for PRS. The effects of the initial site of recurrence were analyzed by the log rank test based on the Kaplan-Meier method. Statistical analyses were performed with SPSS software for Windows, version 12.0 (IBM, Armonk, NY, USA). In both the univariate and the multivariate analyses, $\mathrm{P}<0.05$ was considered to indicate statistical significance.

\section{Results}

Patient demographics are shown in Table 1. The majority of patients had tumors with adenocarcinoma histology (239 cases; $85.1 \%$ ). More than half of the patients (173 cases; $61.6 \%)$ were diagnosed as stage II-III disease at initial surgery. We identified 135 patients $(48.0 \%)$ with EGFR mutations, 33 (11.7\%) with KRAS mutations, 13 (4.7\%) with ALK mutations, and 100 (35.6\%) with triple negative (TN) mutations. One hundred eight of 135 patients $(80.0 \%)$ with EGFR-mutated tumors and 10 of 13 patients $(76.9 \%)$ with ALK-rearranged tumors received TKI therapy after recurrence. Approximately half of the patients received adjuvant therapy $(47.0 \%)$. Definitive local treatment, including radiotherapy and/or surgery, was performed in 92 patients (32.8\%) for recurrence. Among them, metastasectomy was indicated in 34 patients, with resection of 13 pulmonary lesions, four adrenal lesions, and three brain lesions. In terms of the number of the recurrent organs, prevalence of local therapy is $43 \%$ in patients with single organ metastasis, which is higher than that with 
Table 1 Patients characteristics

\begin{tabular}{|c|c|c|}
\hline Variables & $\mathrm{N}=281$ & $\%$ \\
\hline \multicolumn{3}{|l|}{ Sex } \\
\hline Male/female & $161 / 120$ & $57.3 / 42.7$ \\
\hline \multicolumn{3}{|l|}{ Age } \\
\hline Mean \pm SD & $65.3 \pm 9.58$ & - \\
\hline \multicolumn{3}{|l|}{ Smoking history } \\
\hline Present/absent & $163 / 118$ & $58.0 / 42.0$ \\
\hline \multicolumn{3}{|l|}{ Histology } \\
\hline AD/SQ/others & $239 / 16 / 26$ & $85.1 / 5.7 / 9.2$ \\
\hline \multicolumn{3}{|l|}{ Induction therapy } \\
\hline Present & 7 & 2.5 \\
\hline \multicolumn{3}{|l|}{ pStage } \\
\hline$|/| I-|I|$ & $108 / 173$ & $38.4 / 61.6$ \\
\hline \multicolumn{3}{|l|}{ Surgical procedure } \\
\hline Standard/limited & $259 / 22$ & $92.2 / 7.8$ \\
\hline \multicolumn{3}{|l|}{ Oncogenic status } \\
\hline EGFR & 135 & 48.0 \\
\hline ALK & 13 & 4.7 \\
\hline KRAS & 33 & 11.7 \\
\hline TN & 100 & 35.6 \\
\hline \multicolumn{3}{|l|}{ Adjuvant therapy } \\
\hline Present & 132 & 47.0 \\
\hline \multicolumn{3}{|c|}{ Single organ metastasis } \\
\hline Present & 167 & 59.4 \\
\hline \multicolumn{3}{|c|}{ Treatment for recurrence } \\
\hline None & 39 & 13.8 \\
\hline $\mathrm{RT} / \mathrm{S}$ & 37 & 13.2 \\
\hline $\mathrm{CT} / \mathrm{TKI}$ & 150 & 53.4 \\
\hline $\mathrm{RT} / \mathrm{S}+\mathrm{CT} / \mathrm{TKI}$ & 55 & 19.6 \\
\hline \multicolumn{3}{|l|}{ TTR (months) } \\
\hline Mean \pm SD & $12.7 \pm 8.83$ & - \\
\hline
\end{tabular}

$A D$, adenocarcinoma; $S Q$, squamous cell carcinoma; EGFR, epidermal growth factor receptor; ALK, anaplastic lymphoma kinase; KRAS, Kirsten rat sarcoma viral oncogene homolog; $\mathrm{TN}$, triple negative; RT/S, radiotherapy and/or surgery; CT/TKI, chemotherapy and/or tyrosine kinase inhibitor treatment; TTR, time to recurrence. multiple organ metastases $(17.6 \%$; $\mathrm{P}<0.001)$. In patients with single organ metastasis, local therapies were frequently indicated for metastases in the adrenal gland, brain, and locoregional site $(100 \%, 81.5 \%, 63.9 \%$, respectively, data not shown).

The median PRS time among the 281 patients was 26.1 months; the 3 - and 5-year PRS rates were $55.7 \%$ and $34.8 \%$, respectively (Figure $1 A$ ). The median PRS time was 36.1 months for patients with EGFR mutations, 18.2 months for patients with KRAS mutations, 35.8 months for patients with ALK rearrangements, and 16.5 months for patients with TN mutations. The respective 3- and 5-year PRS rates were $68.0 \%$ and $41.9 \%$ for patients with EGFR mutations, $34.1 \%$ and $27.3 \%$ for patients with KRAS mutations, $60.6 \%$ and $60.6 \%$ for patients with ALK rearrangements, and $44.5 \%$ and $24.3 \%$ for patients with $\mathrm{TN}$ mutations $(\mathrm{P}<0.001)$ (Figure $1 B)$.

Univariate analyses were performed to determine factors associated with PRS. Female sex, never having smoked, pStage I, presence of EGFR mutation, single organ metastasis, presence of both local and systemic treatments for recurrence, and longer TTR were significant favorable factors for PRS $(\mathrm{P}=0.026, \mathrm{P}=0.003, \mathrm{P}=0.002$, $\mathrm{P}=0.002, \mathrm{P}=0.013, \mathrm{P}=0.004$, and $\mathrm{P}<0.001$, respectively), Adenocarcinoma histology were not favorable factors for PRS (Table 2). Multivariate analysis revealed that pStage I, presence of EGFR mutation, presence of both local and systemic treatments for recurrence, and longer TTR were independent favorable factors for PRS $(\mathrm{P}=0.011, \mathrm{P}=0.003$, $\mathrm{P}=0.003$, and $\mathrm{P}<0.001$, respectively, Table 3).

Table 4 shows the prevalence rates of initial recurrence at different sites for the whole cohort and for the four oncogenic cohorts. Locoregional sites and lungs were the most frequent metastatic sites. Recurrence in the adrenal glands was observed in only 12 patients (4.3\%). There were significant differences in the prevalence of pleural recurrence among the four groups $(\mathrm{P}=0.043)$; pleural recurrence was most frequent in patients with EGFR mutations (22.2\%). Eleven of the 12 patients (91.7\%) with adrenal gland recurrence had $\mathrm{TN}$ status $(\mathrm{P}=0.001)$. Investigating the effect of the site of initial recurrence on PRS, we found that the presence of single organ metastasis and the presence of pulmonary recurrence prolonged PRS strongly (Figure 2). The 3- and 5-year PRS rates for patients with pulmonary recurrence were favorable at $69.1 \%$ and $50.3 \%$, 


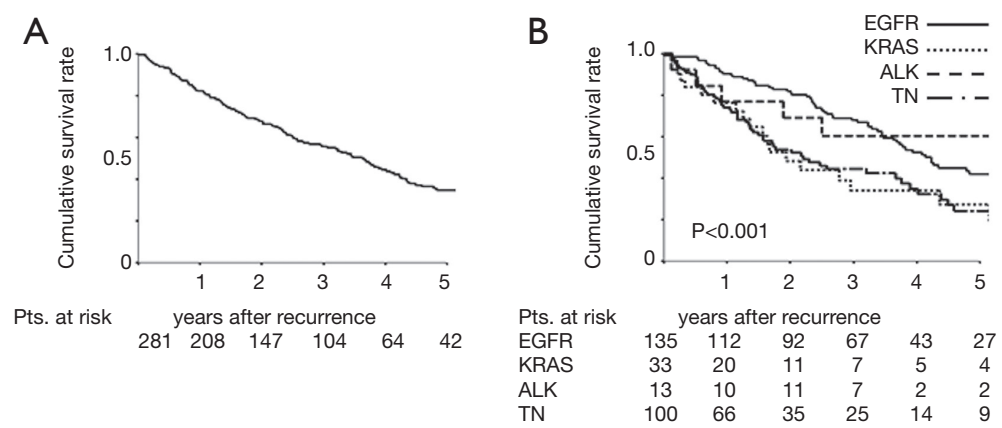

Figure 1 PRS curves of patients with recurrence after resection of NSCLC. (A) PRS curves for all 281 patients with recurrence; (B) PRS curves for patients with recurrence according to oncogenic status. PRS, postrecurrence survival; NSCLC, non-small cell lung cancer.

Table 2 Univariate analyses of factors associated with PRS

\begin{tabular}{|c|c|c|c|c|c|}
\hline Variables & Test & Reference & $\mathrm{HR}$ & $95 \% \mathrm{Cl}$ & $P$ value \\
\hline Age & - & - & 1.013 & $0.995-1.031$ & 0.168 \\
\hline Smoking history & Present & Absent & 1.672 & $1.191-2.348$ & 0.003 \\
\hline Histology & $A D$ & Non-AD & 0.717 & $0.451-1.141$ & 0.161 \\
\hline pStage & I & II-III & 0.573 & $0.471-0.821$ & 0.002 \\
\hline Surgical procedure & Standard & Limited & 1.200 & $0.691-2.084$ & 0.518 \\
\hline \multirow[t]{2}{*}{ Oncogenic status } & EGFR & - & 0.452 & $0.272-0.749$ & 0.002 \\
\hline & ALK & KRAS & 0.422 & $0.158-1.125$ & 0.085 \\
\hline Single organ metastasis & Present & Absent & 0.656 & $0.471-0.915$ & 0.013 \\
\hline \multirow[t]{3}{*}{ Treatment for recurrence } & $\mathrm{RT} / \mathrm{S}$ & None & 0.513 & $0.252-1.043$ & 0.065 \\
\hline & CT/TKI & & 0.643 & $0.375-1.071$ & 0.088 \\
\hline & $\mathrm{RT} / \mathrm{S}+\mathrm{CT} / \mathrm{TKI}$ & & 0.401 & $0.217-0.742$ & 0.004 \\
\hline TTR & - & - & 0.961 & $0.946-0.976$ & $<0.001$ \\
\hline
\end{tabular}

PRS, postrecurrence survival; HR, hazard ratio; Cl, confidence interval; AD, adenocarcinoma; EGFR, epidermal growth factor receptor; ALK, anaplastic lymphoma kinase; KRAS, Kirsten rat sarcoma viral oncogene homolog; TN, triple negative; RT/S, radiotherapy and/or surgery; CT/TKI, chemotherapy and/or tyrosine kinase inhibitor treatment; TTR, time to recurrence.

respectively. On the other hand, the 3- and 5-year PRS rates for patients with recurrence at non-pulmonary sites were $49.8 \%$ and $27.9 \%$, respectively $(\mathrm{P}=0.001)$ (Figure $2 C$ ). The presence of pleural recurrence and/or bone metastasis decreased PRS (Figure 2D,F). The 3- and 5-year PRS rates for the 45 patients with pleural recurrence were extremely low at $32.8 \%$ and $10.2 \%$, respectively $(\mathrm{P}<0.001)$
(Figure 2D). Presence of locoregional recurrence and brain recurrence did not significantly impact PRS (Figure 2B,E).

We divided the cohort into EGFR/ALK and KRAS/ TN groups and evaluated the effects of recurrence site and oncogenic status on PRS. Although the survival of patients with pulmonary recurrence remained high in the EGFR/ ALK group $(\mathrm{P}<0.001 ;$ Figure $3 A)$, the difference of PRS 
Table 3 Multivariate analyses of factors associated with PRS

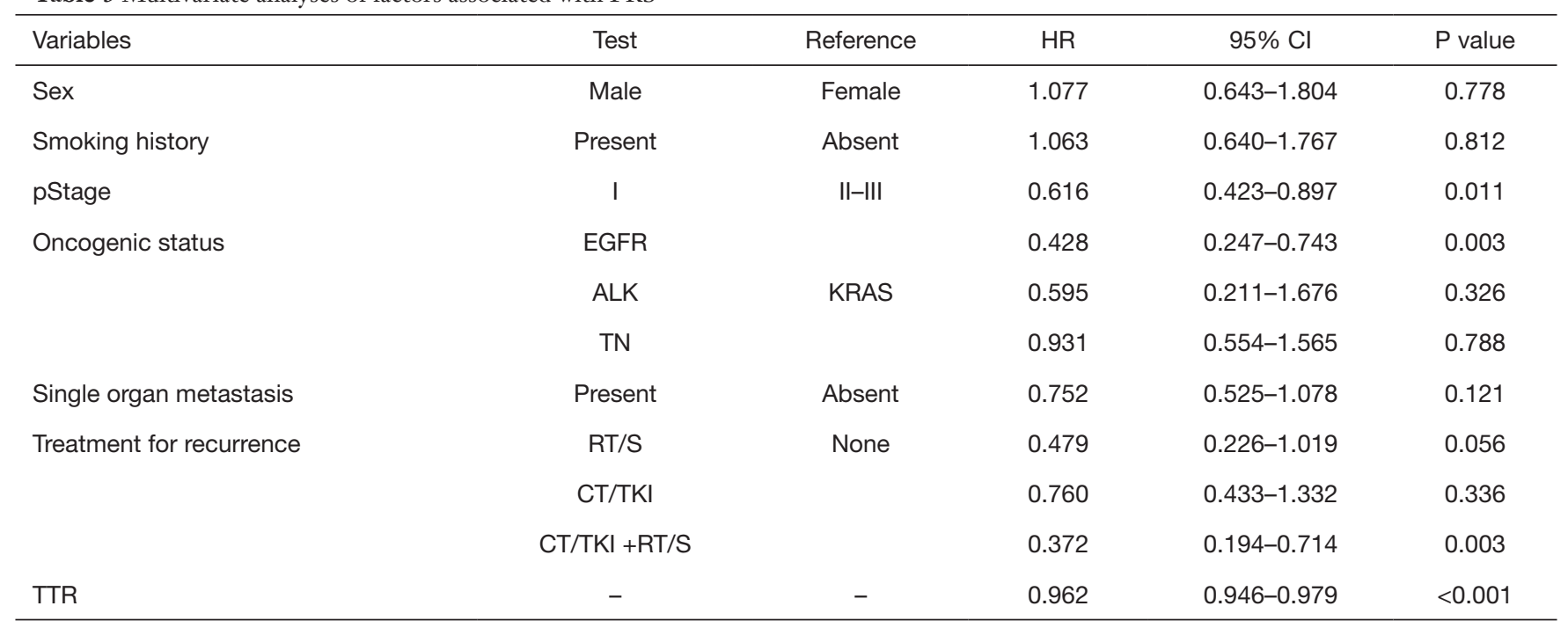

PRS, postrecurrence survival; HR, hazard ratio; CI, confidence interval; EGFR, epidermal growth factor receptor; ALK, anaplastic lymphoma kinase; KRAS, Kirsten rat sarcoma viral oncogene homolog; TN, triple negative; RT/S, radiotherapy and/or surgery; CT/TKI, chemotherapy and/or tyrosine kinase inhibitor treatment; TTR, time to recurrence.

Table 4 Initial sites of recurrence after pulmonary resections by oncogenic status

\begin{tabular}{|c|c|c|c|c|c|c|c|c|c|c|c|}
\hline Recurrent site & \multicolumn{2}{|c|}{ Total } & \multicolumn{2}{|c|}{ EGFR } & \multicolumn{2}{|c|}{ KRAS } & \multicolumn{2}{|c|}{ ALK } & \multicolumn{2}{|c|}{$\mathrm{TN}$} & $P$ value \\
\hline Locoregional site & 83 & 29.5 & 38 & 28.1 & 6 & 18.2 & 5 & 38.5 & 34 & 34.0 & 0.31 \\
\hline Lung & 88 & 31.3 & 43 & 31.9 & 14 & 42.4 & 4 & 30.8 & 27 & 27.0 & 0.64 \\
\hline Pleura & 45 & 16.0 & 30 & 22.2 & 1 & 3.0 & 2 & 15.4 & 12 & 12.0 & 0.043 \\
\hline Bone & 65 & 23.1 & 31 & 23.0 & 10 & 30.3 & 4 & 30.8 & 20 & 20.0 & 0.65 \\
\hline Adrenal gland & 12 & 4.3 & 0 & 0.0 & 1 & 3.0 & 0 & 0.0 & 11 & 11.0 & 0.001 \\
\hline Single organ & 167 & 59.4 & 81 & 60.0 & 19 & 57.6 & 8 & 61.5 & 59 & 59.0 & 0.99 \\
\hline
\end{tabular}

EGFR, epidermal growth factor receptor; ALK, anaplastic lymphoma kinase; KRAS, Kirsten rat sarcoma viral oncogene homolog; TN, triple negative.

was revealed to be unclear in the KRAS/TN group by the presence of pulmonary recurrence $(\mathrm{P}=0.53$; Figure $3 B)$. The presence of pleural recurrence further worsened PRS in both groups $(\mathrm{P}<0.001$ and 0.006 , respectively) (Figure $3 C, D)$. The impact of bone recurrence on PRS was marginal in the EGFR/ALK group $(\mathrm{P}=0.056$, Figure $3 E)$, but not significant in the KRAS/TN group $(\mathrm{P}=0.11$, Figure $3 F)$.

\section{Discussion}

After innovations in diagnostic modalities and treatments, including adjuvant therapy, survival outcomes in patients with resected NSCLC have improved $(1,17,18)$. According to the Japanese Lung Cancer Registry Study, the 5-year survival rate increased to $69.6 \%$ in 2004 from $51.9 \%$ in 1994 (19). Despite improvement in survival, we often observe patients who experience recurrence after surgical resection. The incidence of recurrence varies among reports, ranging from $17.7 \%$ to $41.5 \%(9-12,20)$. The incidence of $27.0 \%$ in our study is consistent with other studies.

The lung, hilar/mediastinal lymph nodes, brain, bone, 

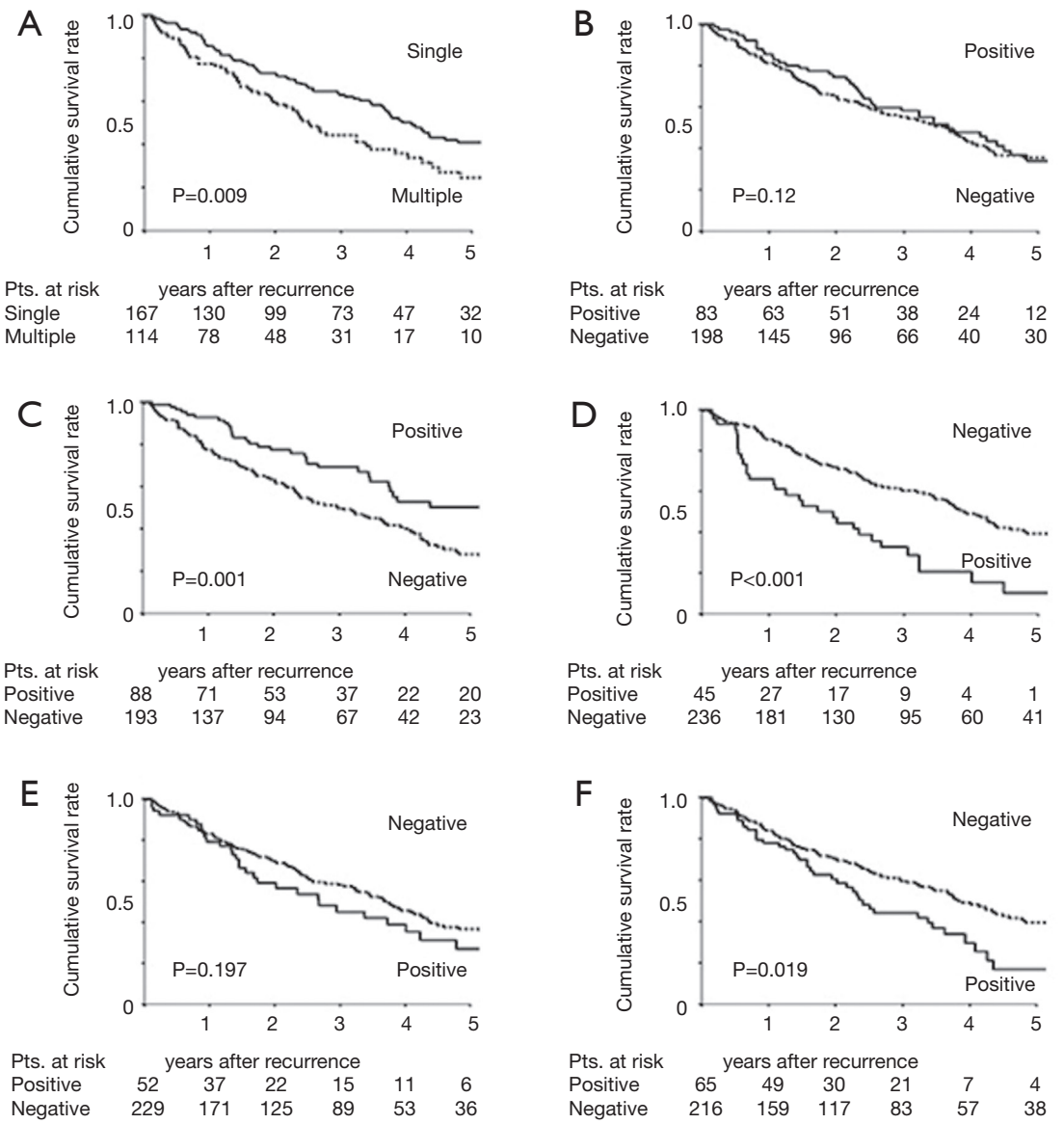

Figure 2 PRS curves of patients with recurrence after resection of NSCLC according to the initial site of recurrence. (A) PRS curves for patients with recurrence at a single organ; (B) PRS curves for patients with locoregional recurrence; (C) PRS curves for patients with pulmonary recurrence; (D) PRS curves for patients with pleural recurrence; (E) PRS curves for patients with brain recurrence; (F) PRS curves for patients with bone recurrence. PRS, postrecurrence survival; NSCLC, non-small cell lung cancer.

liver, pleura, adrenal glands, and others are common metastatic sites of NSCLC. Recurrence can occur in multiple sites as well as single sites. Studies have reported recurrence in multiple sites in $19 \%$ to $24.7 \%$ of patients with recurrence (10-12).

Sonobe et al. reported a $49.1 \%$ recurrence rate in multiple sites in a Japanese cohort, similar to the rate of $40.6 \%$ in our study (9). These results suggest that follow-up policy and diagnostic modalities, in addition to racial issues, may affect patient characteristics.

Survival after recurrence in patients with resected NSCLC is commonly unfavorable. PRS rates of $37 \%$ and $17 \%$ at 1 and 2 years, respectively, were reported by Sugimura et al. (10) and Williams et al. (12). The results of Sugimura et al. and Williams et al. are based on data from patients treated before 2001. Studies based on data including patients whose treatment started in this decade, when molecular targeted therapies were introduced clinically, found better outcomes, with PRS rates of $68.3 \%$ at 1 year and $45.8 \%$ to $52.1 \%$ at 2 years $(20,21)$. Recent analyses of prolonged PRS found 3- and 5-year PRS rates of $28.4-33.0 \%$ and $19.9-29.8 \%$, respectively $(9,22,23)$. Recently, Sekihara et al. reported the novel outcomes of long-term PRS in patients resected between 1993 and 2006 (24), whereas the trend of long-term PRS may not be identical with our cohort. The improvements in PRS may be attributable to several factors, including the introduction of molecular targeted therapies, improved selection of patients for therapy, and earlier detection of recurrence owing to innovative diagnostic modalities. 

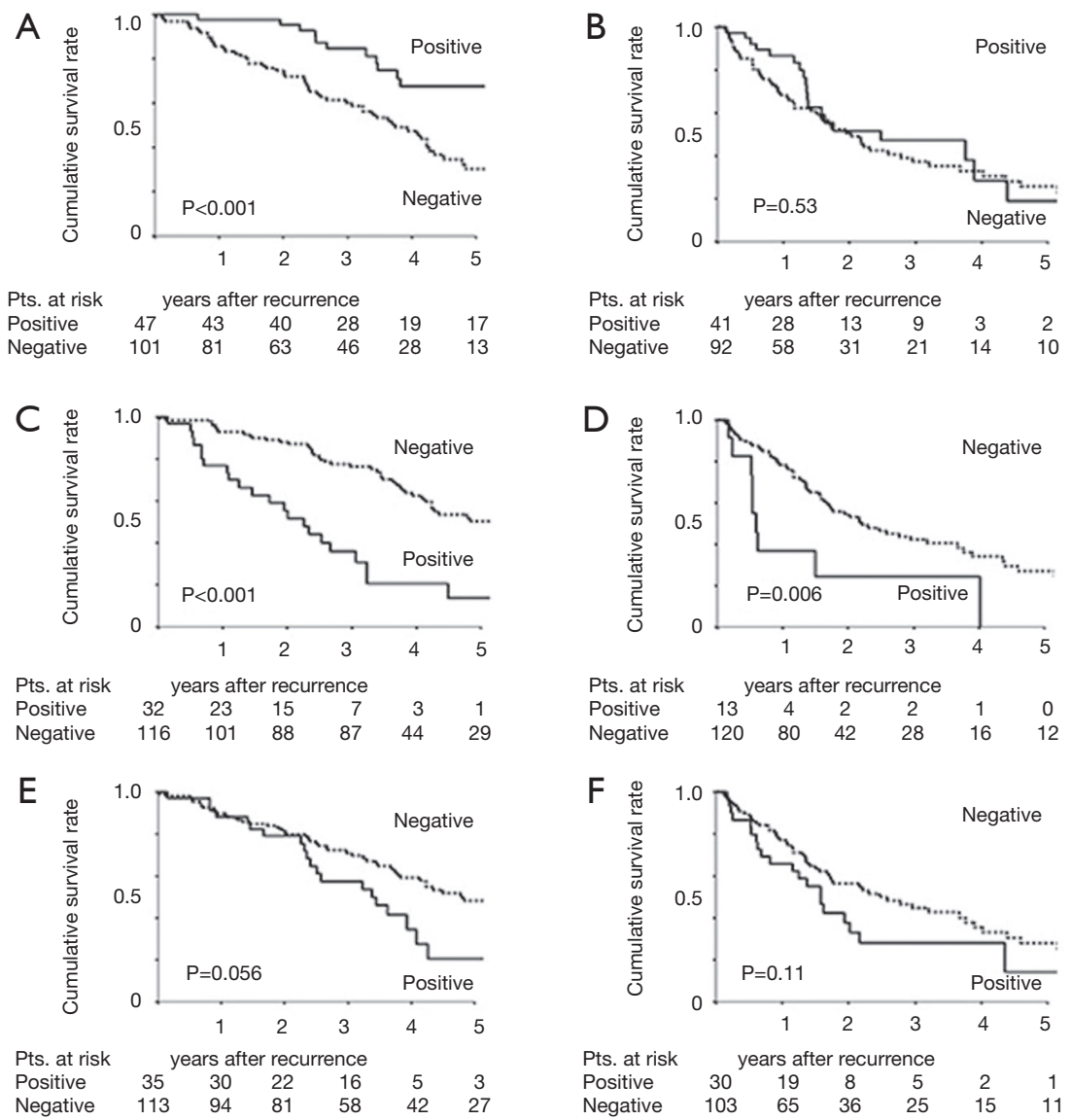

Figure 3 PRS curves of patients with recurrence after resection of NSCLC according to the initial site of recurrence and oncogenic subgroup. (A) PRS curves for patients with EGFR/ALK patients with pulmonary recurrence; (B) PRS curves for patients with KRAS/ TN patients with pulmonary recurrence; (C) PRS curves for EGFR/ALK patients with pleural recurrence; (D) PRS curves for KRAS/TN patients with pleural recurrence; (E) PRS curves for EGFR/ALK patients with bone recurrence; (F) PRS curves for KRAS/TN patients with bone recurrence. PRS, postrecurrence survival; NSCLC, non-small cell lung cancer; EGFR, epidermal growth factor receptor; ALK, anaplastic lymphoma kinase; TN, triple negative.

EGFR-TKIs, including gefitinib and erlotinib, are the targeted drugs that have been introduced into clinical use for treatment of advanced NSCLC. The Iressa Pan-Asia Study showed that clinical factors such as female sex, nonsmoking status, and adenocarcinoma histology predicted the efficacy of gefitinib in East Asia (25). Although whether EGFR gene mutation itself is a prognostic factor is controversial (26-28), the presence of mutations in the kinase domain was strongly correlated with increased response to EGFR-TKI, and more than $70 \%$ of the response rate was prospected. Several randomized phase 3 trials compared gefitinib with the conventional treatment with cisplatin and docetaxel for first-line treatment of advanced NSCLC. These trials demonstrated the superiority of gefitinib treatment for progression-free survival $(14,29)$. In the postoperative recurrence setting, we previously reported the survival benefit of gefitinib treatment in patients with EGFRmutated NSCLC $(3,30)$. New-generation TKIs and treatment with immune checkpoint inhibitors may further improve the prognosis of patients with recurrence in the future.

In this study, we also investigated the effect of the initial site of recurrence on PRS. We found that survival was better in patients with pulmonary recurrence, especially in those with EGFR- or ALK-mutated tumors. Those with pleural recurrence had extremely poor survival. We suggest 
that NSCLC tumors may have a variety of behaviors according to the metastatic sites, and that the sites may be associated with the biological tumor phenotypes. It is possible that tumors associated with pulmonary recurrence originally have a less aggressive nature, making them a good indication for local therapy. Further investigations based on larger series are required to confirm our results concerning the metastatic sites and genetic status.

Our study has several limitations due to the retrospective design. The follow-up policy was not firmly defined; the surveillance interval and the diagnostic modalities were not completely consistent over the study period. Second, the diagnosis of recurrence was mainly based on radiological findings without pathological confirmation. Although we believe that our clinical diagnoses of recurrence were valid, we could not completely rule out that some cases diagnosed as recurrence were cases of metachronous second primary lung cancers. Last, the contributions of driver mutations other than EGFR, ALK, and KRAS were not analyzed. We should also interpret our results considering the heterogeneity of the TN group.

In conclusion, we identified that pStage I, EGFR mutated status, presence of both local and systemic therapies for recurrence, and longer TTR were favorable factors for PRS in patients with resected NSCLC. The beneficial effects of treatment may be related to variables including oncogenic status and metastatic pattern.

\section{Acknowledgements}

None.

\section{Footnote}

Conflict of Interest: The authors have no conflicts of interest to declare.

Ethical Statement: The study was approved by the institutional review board of Aichi Cancer Center (No. 2017-1-243) and waived the need for written informed consent from each patient.

\section{References}

1. Scagliotti GV, Parikh P, von Pawel J, et al. Phase III study comparing cisplatin plus gemcitabine with cisplatin plus pemetrexed in chemotherapy-naive patients with advanced-stage non-small-cell lung cancer. J Clin Oncol
2008;26:3543-51.

2. Lynch TJ, Bell DW, Sordella R, et al. Activating mutations in the epidermal growth factor receptor underlying responsiveness of non-small-cell lung cancer to gefitinib. N Engl J Med 2004;350:2129-39.

3. Mitsudomi T, Kosaka T, Endoh H, et al. Mutations of the epidermal growth factor receptor gene predict prolonged survival after gefitinib treatment in patients with nonsmall-cell lung cancer with postoperative recurrence. J Clin Oncol 2005;23:2513-20.

4. Paez JG, Janne PA, Lee JC, et al. EGFR mutations in lung cancer: correlation with clinical response to gefitinib therapy. Science 2004;304:1497-500.

5. Tamura K, Okamoto I, Kashii T, et al. Multicentre prospective phase II trial of gefitinib for advanced nonsmall cell lung cancer with epidermal growth factor receptor mutations: results of the West Japan Thoracic Oncology Group trial (WJTOG0403). Br J Cancer 2008;98:907-14.

6. Hida T, Nokihara H, Kondo M, et al. Alectinib versus crizotinib in patients with ALK-positive non-small-cell lung cancer (J-ALEX): an open-label, randomised phase 3 trial. Lancet 2017;390:29-39.

7. Shaw AT, Varghese AM, Solomon BJ, et al. Pemetrexedbased chemotherapy in patients with advanced, ALKpositive non-small cell lung cancer. Ann Oncol 2013;24:59-66.

8. Lou F, Sima CS, Rusch VW, et al. Differences in patterns of recurrence in early-stage versus locally advanced nonsmall cell lung cancer. Ann Thorac Surg 2014;98:1755-60; discussion 60-1.

9. Sonobe M, Yamada T, Sato M, et al. Identification of subsets of patients with favorable prognosis after recurrence in completely resected non-small cell lung cancer. Ann Surg Oncol 2014;21:2546-54.

10. Sugimura H, Nichols FC, Yang P, et al. Survival after recurrent nonsmall-cell lung cancer after complete pulmonary resection. Ann Thorac Surg 2007;83:409-17; discussioin 17-8.

11. Taylor MD, Nagji AS, Bhamidipati CM, et al. Tumor recurrence after complete resection for non-small cell lung cancer. Ann Thorac Surg 2012;93:1813-20; discussion 20-1.

12. Williams BA, Sugimura H, Endo C, et al. Predicting postrecurrence survival among completely resected nonsmall-cell lung cancer patients. Ann Thorac Surg 2006;81:1021-7.

13. Hoang T, Xu R, Schiller JH, et al. Clinical model to 
predict survival in chemonaive patients with advanced non-small-cell lung cancer treated with third-generation chemotherapy regimens based on eastern cooperative oncology group data. J Clin Oncol 2005;23:175-83.

14. Mitsudomi T, Morita S, Yatabe Y, et al. Gefitinib versus cisplatin plus docetaxel in patients with non-small-cell lung cancer harbouring mutations of the epidermal growth factor receptor (WJTOG3405): an open label, randomised phase 3 trial. Lancet Oncol 2010;11:121-8.

15. Sekine I, Nokihara H, Yamamoto N, et al. Comparative chemotherapeutic efficacy in non-small cell lung cancer patients with postoperative recurrence and stage IV disease. J Thorac Oncol 2009;4:518-21.

16. Kobayashi Y, Mitsudomi T, Sakao Y, et al. Genetic features of pulmonary adenocarcinoma presenting with groundglass nodules: the differences between nodules with and without growth. Ann Oncol 2015;26:156-61.

17. Douillard JY, Rosell R, De Lena M, et al. Adjuvant vinorelbine plus cisplatin versus observation in patients with completely resected stage IB-IIIA non-small-cell lung cancer (Adjuvant Navelbine International Trialist Association [ANITA]): a randomised controlled trial. Lancet Oncol 2006;7:719-27.

18. Winton T, Livingston R, Johnson D, et al. Vinorelbine plus cisplatin vs. observation in resected non-small-cell lung cancer. N Engl J Med 2005;352:2589-97.

19. Sawabata N, Miyaoka E, Asamura H, et al. Japanese lung cancer registry study of 11,663 surgical cases in 2004: demographic and prognosis changes over decade. J Thorac Oncol 2011;6:1229-35.

20. Saisho S, Yasuda K, Maeda A, et al. Post-recurrence survival of patients with non-small-cell lung cancer after curative resection with or without induction/ adjuvant chemotherapy. Interact Cardiovasc Thorac Surg 2013;16:166-72.

21. Yamazaki K, Sugio K, Yamanaka T, et al. Prognostic factors in non-small cell lung cancer patients with postoperative recurrence following third-generation chemotherapy. Anticancer Res 2010;30:1311-5.

22. Choi PJ, Jeong SS, Yoon SS. Prediction and prognostic factors of post-recurrence survival in recurred patients with early-stage NSCLC who underwent complete resection. J Thorac Dis 2016;8:152-60.

23. Hung JJ, Yeh YC, Jeng WJ, et al. Prognostic Factors of Survival after Recurrence in Patients with Resected Lung Adenocarcinoma. J Thorac Oncol 2015;10:1328-36.

24. Sekihara K, Hishida T, Yoshida J, et al. Long-term survival outcome after postoperative recurrence of non-small-cell lung cancer: who is 'cured' from postoperative recurrence? Eur J Cardiothorac Surg 2017;52:522-8.

25. Mok TS, Wu YL, Thongprasert S, et al. Gefitinib or carboplatin-paclitaxel in pulmonary adenocarcinoma. N Engl J Med 2009;361:947-57.

26. Izar B, Sequist L, Lee M, et al. The impact of EGFR mutation status on outcomes in patients with resected stage I non-small cell lung cancers. Ann Thorac Surg 2013;96:962-8.

27. Kim Y'T, Seong YW, Jung YJ, et al. The presence of mutations in epidermal growth factor receptor gene is not a prognostic factor for long-term outcome after surgical resection of non-small-cell lung cancer. J Thorac Oncol 2013;8:171-8.

28. Kosaka T, Yatabe Y, Onozato R, et al. Prognostic implication of EGFR, KRAS, and TP53 gene mutations in a large cohort of Japanese patients with surgically treated lung adenocarcinoma. J Thorac Oncol 2009;4:22-9.

29. Maemondo M, Inoue A, Kobayashi K, et al. Gefitinib or chemotherapy for non-small-cell lung cancer with mutated EGFR. N Engl J Med 2010;362:2380-8.

30. Katayama T, Matsuo K, Kosaka T, et al. Effect of gefitinib on the survival of patients with recurrence of lung adenocarcinoma after surgery: a retrospective casematching cohort study. Surg Oncol 2010;19:e144-9.
Cite this article as: Mizuno T, Arimura T, Kuroda H, Sakakura N, Yatabe Y, Sakao Y. Current outcomes of postrecurrence survival in patients after resection of non-small cell lung cancer. J Thorac Dis 2018;10(3):1788-1796. doi: 10.21037/ jtd.2018.01.148 